\title{
Influence of Environmental Conditions on c-Jun N-terminal Kinase Mediated Apoptosis of HL60 Cells by Anti-Cancer Drugs
}

\author{
Eun-Hye Hur, Mun Jung Kang, Sung-Doo KIm, Sung-Nam Lim, Dae-Young KIm, \\ Jung-Hee LeE, Kyoo-Hyung LeE, and Je-Hwan LEE* \\ Department of Hematology, Asan Medical Center, University of Ulsan College of Medicine, Seoul 138-736, Republic of Korea
}

(Received December 30, 2009; Revised January 11, 2010; Accepted January 11, 2010)

\begin{abstract}
Activation of JNK has long been associated with the apoptotic response induced by various anti-cancer drugs including doxorubicin, vinblastine, and etoposide. In this study, we examined and compared patterns of apoptosis and JNK activation according to three different anti-cancer drugs (daunorubicin, vinblastine, and etoposide) and two different sources of HL60 cells (Jackson Laboratory and ATCC). HL60 cells from Jackson Laboratory (HL60/RPMI) were maintained in RPMI 1640 containing $5 \%$ fetal bovine serum and those from ATCC (HL60/IMDM) in IMDM containing 20\% fetal bovine serum as to each manufacture's guideline. In general, HL60/RPMI cells were more sensitive to anti-cancer drugs compared to HL60/IMDM cells, demonstrated by the XTT and flow cytometric analyses. Apoptotic pathways after treatment with anti-cancer drugs seemed to be different between HL60/RPMI (daunorubicin and etoposide, caspase 3 dependent, but caspase 8 or 9 independent; vinblastine, caspase 3 independent) and HL60/IMDM (caspase 3 and caspase 9 dependent). The expression of apoptotic protein, BID, was consistent with caspase 3 activation. Immunoblotting of phospho-JNK and JNK kinase assay showed JNK activation by all three anti-cancer drugs in HL60/RPMI, while JNK activation was observed only in vinblastine-treated cells in HL60/IMDM. Our study results suggest that in vitro environmental conditions have a significant influence on JNK mediated apoptosis of HL60 cells by anti-cancer drugs and in vitro culture conditions are important factors in JNK or possibly other MAPK related studies.
\end{abstract}

Keywords: JNK, Anticancer drugs, HL60

\section{INTRODUCTION}

C-Jun N-terminal kinase (JNK), also known as stress-activated MAP kinases (SAPK), represents one of three groups of mitogen-activated protein kinases (MAPK) that are involved in the regulation of cell proliferation, differentiation, and apoptosis (Davis, 2000; Nishina et al., 2004; Weston and Davis, 2007; Dhanasekaran and Reddy, 2008). JNK is a key regulator of many cellular events and has pro- or anti-apoptotic functions. Whether the activation of JNK leads to cell proliferation or apoptosis depends on cell type, nature of death stimulus, duration of its activation and the activity of other signaling pathway (Lin and Dibling, 2002; Liu and Lin, 2005; Dhanasekaran and Reddy, 2008). JNK can be activated by several different stimuli including

${ }^{*}$ Corresponding author

Tel: +82-2-3010-3218 Fax: +82-2-3010-6961

E-mail: jhlee3@amc.seoul.kr growth factors, cytokines, and stress factors such as ultraviolet irradiation, heat shock, osmotic shock, and many cytotoxic or genotoxic agents (Davis, 2000; Weston and Davis, 2007; Dhanasekaran and Reddy, 2008). Multiple splice variants of JNKs encoded by three distinct genes, namely JNK1, JNK2, and JNK3, have been identified (Davis, 2000; Johnson and Nakamura, 2007). JNK1 and JNk2 are ubiquitously expressed, but the expression of JNK3 is mainly restricted to brain, heart, and testis. There are different substrate specificities among isoforms; therefore, different JNK isoforms may play specific roles, but there is also complementation between the JNK genes (Davis, 2000; Cui et al., 2007).

Anti-cancer drugs are considered to mediate cell death by activating key elements of the apoptosis program and the cellular stress response (Herr and Debatin, 2001). MAPK signaling pathways have been implicated in the response of tumor cell to anti-cancer drugs (Fan and 
Chambers, 2001; Boldt et al., 2002). Among the pathways, activation of JNK has long been associated with the apoptotic response induced by various anti-cancer drugs including doxorubicin (Osborn and Chambers, 1996; Yu et al., 1996; Brantley-Finley et al., 2003; Panaretakis et al., 2005; Lagadinou et al., 2008), vinblastine (Osborn and Chambers, 1996; Fan et al., 2001; Brantley-Finley et al., 2003; Kolomeichuk et al., 2008), etoposide (Osborn and Chambers, 1996; Brantley-Finley et al., 2003), cytarabine (Sampath and Plunkett, 2000; Sampath et al., 2006), and other agents (Choi and Lee, 2009). However, opposing functions of JNK have also been evidenced for anti-cancer drugs. JNK may play a protective role in some situations (Potapova et al., 1997; Wisdom et al., 1999), and inhibition of JNK2 expression was shown to induce apoptosis of human tumor cell (Potapova et al., 2000). In acute myeloid leukemia cells, multidrug resistance has been related to JNK activity (Potapova et al., 2000; Cripe et al., 2002; Do et al., 2007). Cytarabine induced apoptosis in HL60 cells was preceded by the activation of JNK, but the inhibition of JNK activation did not block apoptosis of HL60 cells by cytarabine (Stadheim et al., 2000). Thus, JNK signaling may induce apoptosis or promote cell survival and proliferation after treatment with anti-cancer drugs and may be dispensable for apoptosis. The effects of JNK on cellular responses to anti-cancer drugs appear to depend on the cell type, the class of anti-cancer drugs, the context of other signals received by the cell, and environmental conditions. We have studied the role of JNK activation on the effects of anti-cancer drugs and found different patterns of apoptosis according to different anti-cancer drugs and different sources of the same cells (HL60). Our results indicate that in vitro environmental conditions are important in the studies of JNK mediated apoptosis by anti-cancer drugs.

\section{MATERIALS AND METHODS}

\section{Reagents}

All of three anti-cancer drugs were used for the study: daunorubicin, vinblastine, and etoposide (Sigma-Aldrich, Saint Louis, MO). The drugs are common anti-leukemic agents and the main mechanism of action is inhibition of topoisomerase II in daunorubicin and etoposide, and inhibition of the assembly of microtubules in vinblastine. For the concentrations of the agents in this study, we made reference to a prior study, which investigated JNK activation by anti-cancer drugs (Brantley-Finley et al., 2003). Primary antibodies for the immunoblot assays, which were against human antigens, and Chaps Cell Extract Buffer were purchased from Cell Signaling Technology (Danvers, MA).
The sodium 3'-[1-(phenylaminocarbonyl)-3,4-tetrazolium]bis (4-methoxy-6-nitro) benzene sulfonic acid hydrate (XTT) assay was performed with Cell Proliferation Kit II (Roche Applied Science, Switzerland).

\section{Cell lines and culture media}

Two difference sources of HL60 cells were used for the study: HL60 cells from ATCC (Manassas, VA), which were maintained in Iscove's Modified Dulbecco's Medium (IMDM; ATCC) containing 20\% fetal bovine serum and $1 \%$ L-glutamine, and those from the Jackson Laboratory (Sacramento, CA), which were maintained in RPMI Medium 1640 (Invitrogen, Carlsbad, CA) containing 5\% fetal bovine serum and $1 \% \mathrm{~L}$-glutamine. The cells were passaged twice a week.

\section{Assays for cellular viability}

Cellular viability was evaluated using the XTT assay and flow cytometric analysis. The XTT assay is based on the cleavage of the yellow tetrazolium salt XTT to form an orange formazan dye by metabolically active viable cells (Gerlier and Thomasset, 1986; Jost et al., 1992). For the assay, $1.5 \times 10^{3} \mathrm{HL} 60$ cells were split onto each well of 96-well microplates. The XTT labeling mixture was added 72 hours after treatment of each anti-cancer drug and the microplates were incubated for 4 hours at $37^{\circ} \mathrm{C}$. The absorbance was measured using an ELISA reader at $480 \mathrm{~nm}$ with a reference wave-length at $650 \mathrm{~nm}$.

For flow cytometric analysis of cellular viability (Krishan, 1975), $1 \times 10^{6}$ cells re-suspended in $1.5 \mathrm{ml}$ complete medium were put in a $15 \mathrm{ml}$ polypropylene tube and $1.5 \mathrm{ml} \mathrm{se}$ rum free medium including each anti-cancer drug was added. Twenty-four hours later, the cells were fixed with cold ethanol $(70 \%)$ overnight at $4{ }^{\circ} \mathrm{C}$, and were stained with $60 \mathrm{mcg} / \mathrm{ml}$ propidium iodide (PI; Sigma-Aldrich) containing 10 units $/ \mathrm{ml}$ RNase ONE (Promega, Madison, WI) for 30 minutes. The cells were incubated at $4^{\circ} \mathrm{C}$ until analysis. The percentages of $\mathrm{PI}$ stained cells were measured using a fluorescence-activated cell sorting flow cytometer (Becton Dickinson, San Jose, CA) and inbuilt software.

\section{JNK assay and immunoblot analysis}

Total JNK protein and activated phosphor-JNK protein were assayed with immunoblot analysis. The cells were lysed with Chaps Cell Extract Buffer and protein concentration was determined using a Protein Assay Reagent $A$ (Bio-Rad Laboratories, Hercules, CA). The cell lysates (50 $\mathrm{mcg}$ ) were fractionated by SDS-PAGE, and were transferred to nitrocellulose membrane by BBlot $^{\mathrm{TM}}$ Dry Blotting System (Invitrogen). Membranes were blocked in 5\% skim 
milk in TBST buffer and were incubated overnight with anti-JNK or anti-phospho-JNK antibodies (Thr183/Tyr185) at $4^{\circ} \mathrm{C}$. The blots were visualized with SuperSignal West Pico Chemiluminescent Substrate (Thermo Scientific, Waltham,
MA). Immunoblot analyses were also performed using other primary antibodies for caspases, PARP, Bid, Bax, acetylated histone $H 3$, survivin, and actin with the same protocol.
A

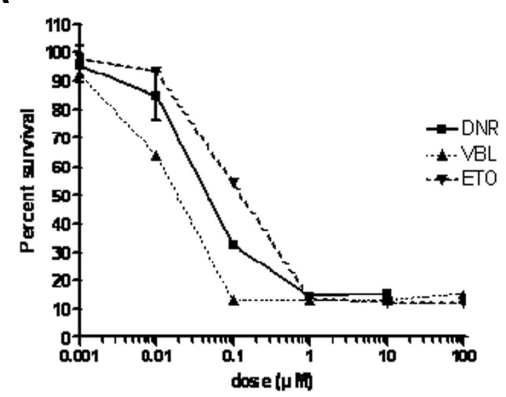

\begin{tabular}{|l|c|}
\hline \multicolumn{2}{|c|}{$\mathrm{IC}_{50}(\mu \mathrm{M}) / \mathrm{RPMI}$} \\
\hline $\mathrm{DNR}$ & 0.04 \\
\hline VBL & 0.02 \\
\hline ETO & 0.2 \\
\hline
\end{tabular}

HL60/RPMI

B

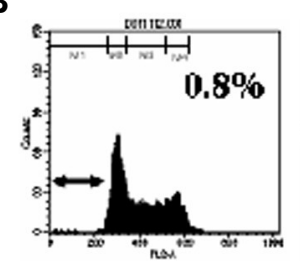

HL60/IVIDIVI

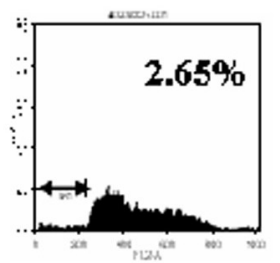

C

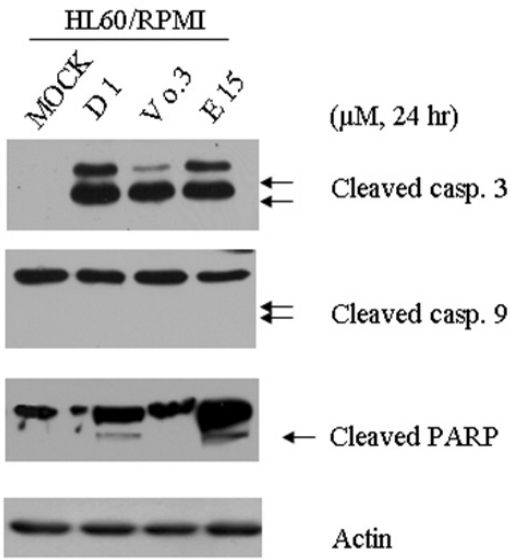

DNR 1 M

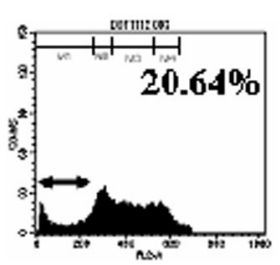

DNR $1 \mu M$
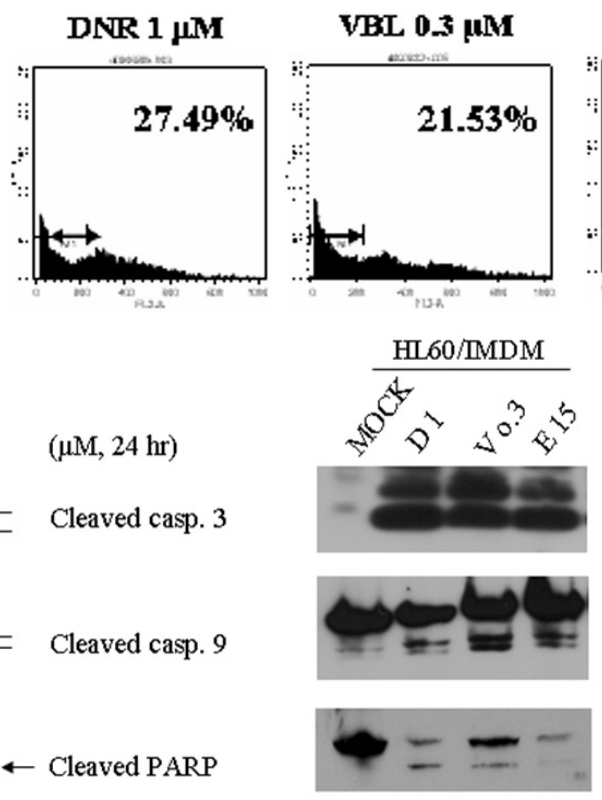

$(\mu \mathrm{M}, 24 \mathrm{hr})$

$\leftarrow$ Cleaved casp. 3

$\leftleftarrows$ Cleaved casp. 9

Cleaved PARP

Actin
Fig. 1. Cellular viability and caspase activation after treatment of anti-cancer drugs on two different sources of HL60 cells (HL60/RPMI and HL60/IMDM). DNR: daunorubicin, VBL: vinblastine, ETO: etoposide. (A) XTT assay. The IC50 values for daunorubicin, vinblastine, and etoposide were $0.04 \mu \mathrm{M}, 0.02 \mu \mathrm{M}$, and 0.2 $\mu \mathrm{M}$ in HL60/RPMI, while those were 0.2 $\mu \mathrm{M}, 0.1 \mu \mathrm{M}$, and $3 \mu \mathrm{M}$ in HL60/IDMD. (B) Flow cytometric analysis with PI staining. The percent proportions of apoptotic cells 24 hours after treatment of anti-cancer drugs are presented. (C) Caspase pathway activation. 
JNK kinase assay was performed 24 hours after treatment of anti-cancer drugs. After the cells were rinsed with ice-cold PBS, $0.5 \mathrm{ml}$ ice-cold 1X Cell Lysis Buffer (Cell Signaling Technology) and $1 \mathrm{mM}$ phenylmethylsulfonyl fluoride were added, and procedure of freezing and thawing was repeated three times. The lysates were centrifuged at $4^{\circ} \mathrm{C}, 14,000 \mathrm{rpm}$ for 10 minutes and the supernatants were adjusted to $500 \mathrm{mcg}$ of protein in $0.5 \mathrm{ml}$, then stored at $-80^{\circ} \mathrm{C}$. For immunoprecipitation with immobilized c-Jun substrate, $0.5 \mathrm{ml}$ cell lysates were incubated with $500 \mathrm{mcg}$ GST-C-Jun bead slurry (kindly provided by prof. E.J. Choi,
Korea University) overnight at $4^{\circ} \mathrm{C}$. The immunoprecipitates were centrifuged at $4^{\circ} \mathrm{C}, 5,000 \mathrm{rpm}$ for $3 \mathrm{~min}$ to removed supernatant and washed two times with $500 \mathrm{mcl} 1 \mathrm{X}$ Cell Lysis Buffer, then washed two times with $500 \mathrm{mcl} 1 \mathrm{X}$ Kinase Buffer (Cell Signaling Technology). The washed immunoprecipitates were suspended in $50 \mathrm{mcl}$ of $1 \mathrm{X}$ Kinase Buffer supplemented with $200 \mathrm{mcM}$ ATP and incubated at $30^{\circ} \mathrm{C}$ for $30 \mathrm{~min}$. The reaction was stopped by addition with $20 \mathrm{mcl} 2 \mathrm{X}$ SDS Sample Buffer (Bio-Rad Laboratories). The lysates were heated in a boiling water bath for $5 \mathrm{~min}$ and subsequently subjected to SDS-PAGE
A

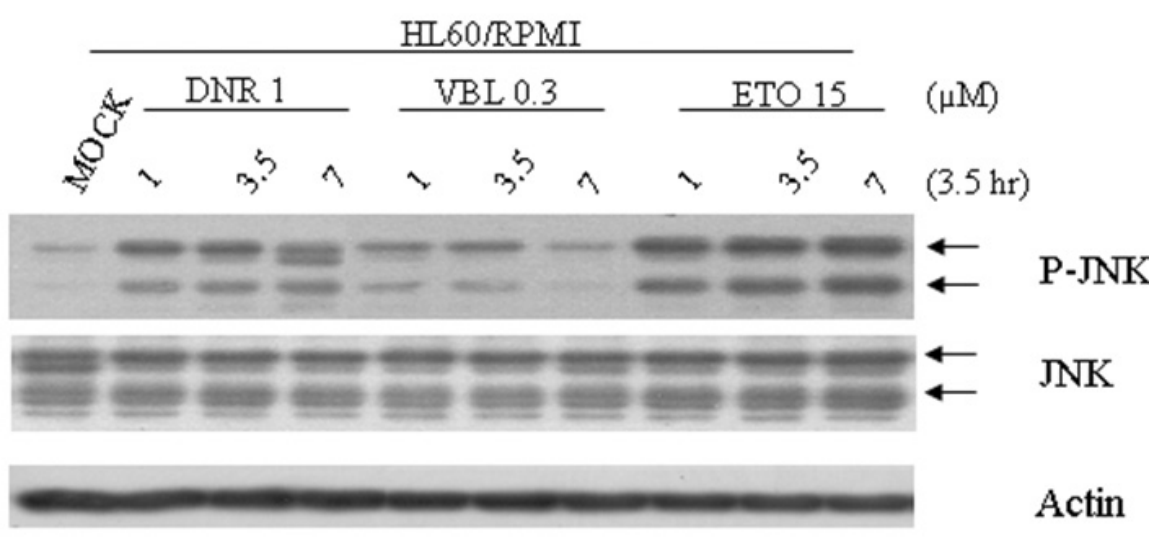

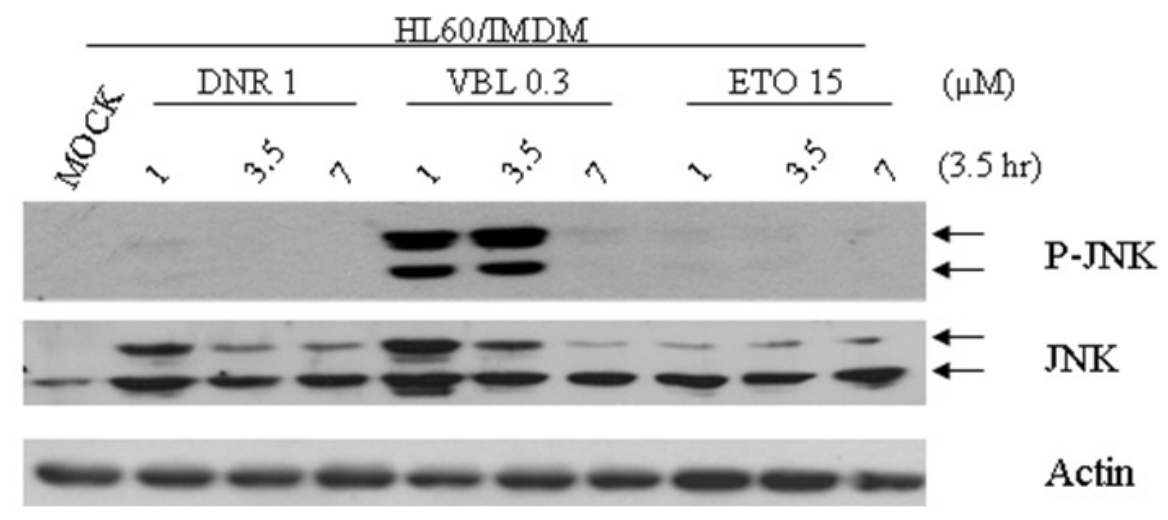

B

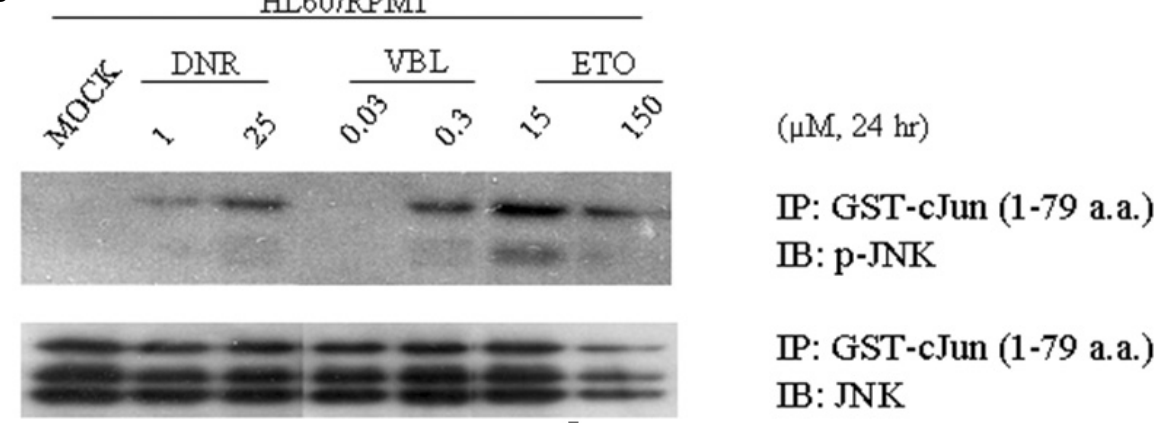

Fig. 2. Activation of JNK by anti-cancer drugs. DNR: daunorubicin, VBL: vinblastine, ETO: etoposide. (A) Changes of intracellular JNK and phospho-JNK amounts $1,3.5$, and 7 hours after treatment of anti-cancer drugs on HL60/RPMI and HL60/IMDM. Of double bands, upper band is for JNK2 $(55 \mathrm{KD})$ and lower one is for JNK1/3 (46 KD). (B) Results of JNK kinase assay after treatment of anti-cancer drugs on HL60/RPMI. IP: immunoprecipitation, IB: immunoblotting. 
on a $12 \%$ polyacrylamide gel, then followed by immunoblot analysis.

\section{RESULTS}

\section{Cellular viability after the treatment of anti-cancer drugs on two different sources of HL60 cells}

Sensitivity to anti-cancer drugs was different between HL60 cells from ATCC (HL60/IMDM) and those from Jackson Laboratory (HL60/RPMI) (Fig. 1). The cellular proliferation rates 72 hours after treatment with anti-cancer drugs were evaluated with the XTT assay, which indicated that the IC50 values for daunorubicin, vinblastine, and etoposide were lower in HL60/RPMI than in HL60/IMDM (0.04 $\mathrm{mcM}$ vs $0.2 \mathrm{mcM}$ for daunorubicin, $0.02 \mathrm{mcM}$ vs. $0.1 \mathrm{mcM}$ for vinblastine, and $0.2 \mathrm{mcM}$ vs. $3 \mathrm{mcM}$ for etoposide) (Fig. $1 \mathrm{~A})$. The results mean that HL60/RPMI has higher sensitivity to anti-cancer drugs than HL60/IMDM. Flow cytometric analysis with PI staining 24 hours after treatment with anti-cancer drugs showed similar findings to the XTT assay (Fig. 1B). The proportion of apoptotic cells after etoposide treatment was much higher in HL60/RPMI than in HL60/IMDM (52.4\% vs. $20.4 \%)$. Caspases play a crucial role in the execution of the apoptotic process (Philchenkov, 2004; Lavrik et al., 2005). We observed different patterns of caspase activation after treatment of anti-cancer drugs between HL60/RPMI and HL60/IMDM (Fig. 1C). In HL60/ RPMI, daunorubicin and etoposide caused the activation of caspase 3 and poly(ADP-ribose) polymerase (PARP), but vinblastine did not. In HL60/IMDM, however, all three drugs caused the activation of caspase 3 and PARP. Cleavage of caspase 9 by anti-cancer drugs was observed only in HL60/IMDM (not in HL60/RPMI), while caspase 8 was not activated both in HL60/RPMI and HL60/IMDM.

\section{Activation of JNK by anti-cancer drugs}

We observed the changes of intracellular JNK and phospho-JNK amounts 1, 3.5, and 7 hours after treatment of anti-cancer drugs (Fig. 2A). Intracellular amounts of total JNK protein did not change after treatment of anti-cancer drugs in HL60/IMDM, while amounts of JNK proteins in HL60/IMDM increased after treatment of anti-cancer drugs. Regarding JNK isoforms, amounts of JNK1/3 and JNK2 were similar in HL60/RPMI, while amount of JNK2 was less than JNK1/3 amount in HL60/IMDM. The patterns of JNK activation, measured by phospho-JNK, were different according to anti-cancer drugs and sources of cells. In HL60/RPMI, JNK was well activated after treatment of all three anti-cancer drugs although vinblastine induced acti-

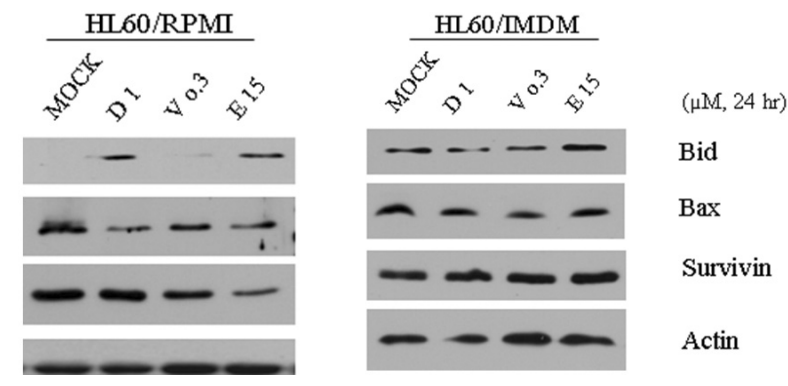

Fig. 3. Expression of apoptotic proteins by anti-cancer drugs. D: daunorubicin, $\mathrm{V}$ : vinblastine, $\mathrm{E}$ : etoposide.

vation of JNK decreased more rapidly than daunorubicin or etoposide induced activation. In HL60/IMDM, only vinblastine caused activation of JNK. JNK kinase assay also showed increase of JNK kinase activity after treatment of each anti-cancer drug in HL60/RPMI (Fig. 2A).

\section{Expression of apoptotic proteins by anti-cancer drugs}

$\mathrm{BH} 3$ interacting domain death agonist (BID) and $\mathrm{Bcl}-2$ associated $X$ protein (Bax) are pro-apoptotic members of the $\mathrm{Bcl}-2$ protein family. BID expression patterns were correlated with activation patterns of caspase 3 . In HL60/ RPMI, BID expression was upregulated by daunorubicin or etoposide, but not by vinblastine, while Bax expression was not changed after treatment of anti-cancer drugs (Fig. 3). In HL60/IMDM, BID or Bax was constitutively expressed and was not changed by anti-cancer drugs. Survivin is a member of the inhibitor of apoptosis family and the survivin protein functions to inhibit caspase activation. Survivin expression decreased in HL60/RPMI by etoposide, but it was not changed in other situations.

\section{DISCUSSION}

In this study, we examined and compared patterns of apoptosis and JNK activation according to three different anti-cancer drugs (daunorubicin, vinblastine, and etoposide) and two different sources of the same cells (HL60/RPMI and HL60/IMDM). HL60 cells of each source were maintained with different culture media that were recommended by each manufacture's guideline. Surprisingly, patterns of apoptosis and JNK activation by anti-cancer drugs were significantly different between two sources of cells. In general, HL60/RPMl cells were more sensitive to anti-cancer drugs compared to HL60/IMDM cells, demonstrated by the XTT and flow cytometric analyses. Apoptotic pathways after treatment with anti-cancer drugs seemed to be different between HL60/RPMI (daunorubicin and etoposide, caspase 3 dependent, but caspase 8 or 9 in- 
dependent; vinblastine, caspase 3 independent) and HL60/IMDM (caspase 3 and caspase 9 dependent). The anti-cancer drugs that were used in this study have been known to induce apoptosis, at least in part, through caspase 3 activation (Gamen et al., 1997; Benjamin et al., 1998; Tashiro et al., 1998; Turnbull et al., 1999). In this study, however, we could not find caspase 3 activation after vinblastine treatment in HL60/RPMl cells and expression of apoptotic protein, BID, was consistent with caspase 3 activation. The findings indicate that diverse apoptotic pathways are present in response to anti-cancer drug (Herr and Debatin, 2001) and there may be different cellular responses according to status of the cells even though the same anti-cancer drug (vinblastine in this study) is treated to the same cell type (HL60 cells in this study). Previous studies have shown that anti-cancer drugs induce the activation of JNK pathway, which may contribute to apoptosis or proliferation of cells (Fan and Chambers, 2001; Herr and Debatin, 2001; Boldt et al., 2002). Immunoblotting of phospho-JNK and JNK kinase assay showed JNK activation by all three anti-cancer drugs in HL60/RPMI, while JNK activation was observed only in vinblastine-treated cells in HL60/IMDM. The findings suggest that the role of JNK pathway in the apoptosis after treatment with anti-cancer drugs may be various according to the cell type, nature of anti-cancer drug, and cellular status (Fan and Chambers, 2001; Herr and Debatin, 2001; Boldt et al., 2002; Brantley-Finley et al., 2003) The various patterns of apoptosis and JNK activation by anti-cancer drugs in this study may be originated from different in vitro environmental conditions between two sources of cells, not from source itself. There was difference in the concentration of fetal bovine serum in the culture media; $5 \%$ in HL60/RPMI and 20\% in HL60/IMDM. Fetal bovine serum contains a variety of growth factors and cellular response to anti-cancer drugs may be affected by the concentration of fetal bovine serum in the media. In addition, RPMI and IMDM may provide different environmental conditions in cell culture system.

In conclusion, our study results suggest that in vitro environmental conditions have a significant influence on JNK mediated apoptosis of HL60 cells by anti-cancer drugs and in vitro culture conditions are important factors in JNK or possibly other MAPK related studies.

\section{ACKNOWLEDGMENTS}

This research was supported by the Converging Research Center Program through the National Research Foundation of Korea (NRF) funded by the Ministry of
Education, Science and Technology (KRF-2005-041E00190).

\section{REFERENCES}

Benjamin, C. W., Hiebsch, R. R. and Jones, D. A. (1998). Caspase activation in MCF7 cells responding to etoposide treatment. Mol. Pharmacol. 53, 446-450.

Boldt, S., Weidle, U. H. and Kolch, W. (2002). The role of MAPK pathways in the action of chemotherapeutic drugs. Carcinogenesis. 23, 1831-1838.

Brantley-Finley, C., Lyle, C. S., Du, L., Goodwin, M. E., Hall, T., Szwedo, D., Kaushal, G. P. and Chambers, T. C. (2003) The JNK, ERK and p53 pathways play distinct roles in apoptosis mediated by the antitumor agents vinblastine, doxorubicin, and etoposide. Biochem. Pharmacol. 66, 459-469.

Choi, J. H. and Lee, K. T. (2009). Costunolide-induced apoptosis in human leukemia cells: involvement of $\mathrm{c}$-jun N-terminal kinase activation. Biol. Pharm. Bull. 32, 18031808.

Cripe, L. D., Gelfanov, V. M., Smith, E. A., Spigel, D. R., Phillips, C. A., Gabig, T. G., Jung, S. H., Fyffe, J., Hartman, A. D., Kneebone, P., Mercola, D., Burgess, G. S. and Boswell, H. $\mathrm{S}$. (2002). Role for c-jun N-terminal kinase in treatmentrefractory acute myeloid leukemia (AML): signaling to multidrug-efflux and hyperproliferation. Leukemia 16, 799812.

Cui, J., Zhang, M., Zhang, Y.Q. \& Xu, Z. H. (2007). JNK pathway: diseases and therapeutic potential. Acta Pharmacol. Sin. 28, 601-608.

Davis, R. J. (2000). Signal transduction by the JNK group of MAP kinases. Cell 103, 239-252.

Dhanasekaran, D. N. and Reddy, E. P. (2008). JNK signaling in apoptosis. Oncogene 27, 6245-6251.

Do, J. H., Oh, S. H., Song, E. J., Chung, J. S., Kang, C. D. and Lee, E. Y. (2007). Treatment outcome of multidrug resistance related mRNA expression and $\mathrm{c}$-jun-N-terminal kinase activity in patients with acute myeloid leukemia. Korean $\mathrm{J}$. Lab. Med. 27, 229-236.

Fan, M. and Chambers, T. C. (2001). Role of mitogen-activated protein kinases in the response of tumor cells to chemotherapy. Drug Resist. Updat. 4, 253-267.

Fan, M., Goodwin, M. E., Birrer, M. J. \& Chambers, T. C. (2001). The c-Jun $\mathrm{NH}(2)$-terminal protein kinase/AP-1 pathway is required for efficient apoptosis induced by vinblastine. Cancer Res. 61, 4450-4458.

Gamen, S., Anel, A., Lasierra, P., Alava, M. A., MartinezLorenzo, M. J., Pineiro, A. and Naval, J. (1997). Doxorubicininduced apoptosis in human T-cell leukemia is mediated by caspase-3 activation in a Fas-independent way. FEBS Lett. 417, 360-364.

Gerlier, D. and Thomasset, N. (1986). Use of MTT colorimetric assay to measure cell activation. J. Immunol. Methods 94, 57-63.

Herr, I. and Debatin, K.M. (2001). Cellular stress response and apoptosis in cancer therapy. Blood 98, 2603-2614.

Johnson, G. L. and Nakamura, K. (2007). The c-jun kinase/ stress- activated pathway: regulation, function and role in human disease. Biochim. Biophys. Acta 1773, 1341-1348. 
Jost, L. M., Kirkwood, J. M. and Whiteside, T. L. (1992) Improved short- and long-term XTT-based colorimetric cellular cytotoxicity assay for melanoma and other tumor cells. J. Immunol. Methods 147, 153-165.

Kolomeichuk, S. N., Bene, A., Upreti, M., Dennis, R. A., Lyle, C. S., Rajasekaran, M. \& Chambers, T. C. (2008). Induction of apoptosis by vinblastine via c-Jun autoamplification and p53-independent down-regulation of p21WAF1/CIP1. Mol. Pharmacol. 73, 128-136.

Krishan, A. (1975). Rapid flow cytofluorometric analysis of mammalian cell cycle by propidium iodide staining. J. Cell. Biol. 66, 188-193.

Lagadinou, E. D., Ziros, P. G., Tsopra, O. A., Dimas, K., Kokkinou, D., Thanopoulou, E., Karakantza, M., Pantazis, P., Spyridonidis, A. and Zoumbos, N. C. (2008). c-Jun $\mathrm{N}$-terminal kinase activation failure is a new mechanism of anthracycline resistance in acute myeloid leukemia. Leukemia 22, 1899-1908.

Lavrik, I. N., Golks, A. and Krammer, P. H. (2005). Caspases: pharmacological manipulation of cell death. J. Clin. Invest. 115, 2665-2672.

Lin, A. and Dibling, B. (2002). The true face of JNK activation in apoptosis. Aging Cell 1, 112-116.

Liu, J. and Lin, A. (2005). Role of JNK activation in apoptosis: a double-edged sword. Cell Res. 15, 36-42.

Nishina, H., Wada, T. and Katada, T. (2004). Physiological roles of SAPK/JNK signaling pathway. J. Biochem. 136, 123-126.

Osborn, M.T. and Chambers, T.C. (1996). Role of the stressactivated/c-Jun $\mathrm{NH} 2$-terminal protein kinase pathway in the cellular response to adriamycin and other chemotherapeutic drugs. J. Biol. Chem. 271, 30950-30955.

Panaretakis, T., Laane, E., Pokrovskaja, K., Bjorklund, A. C., Moustakas, A., Zhivotovsky, B., Heyman, M., Shoshan, M. C. and Grander, D. (2005). Doxorubicin requires the sequential activation of caspase-2, protein kinase Cdelta, and c-Jun $\mathrm{NH} 2$-terminal kinase to induce apoptosis. Mol. Biol. Cell. 16, 3821-3831.

Philchenkov, A. (2004). Caspases: potential targets for regulating cell death. J. Cell. Mol. Med. 8, 432-444.

Potapova, O., Gorospe, M., Dougherty, R. H., Dean, N. M.,
Gaarde, W. A. and Holbrook, N. J. (2000). Inhibition of C-Jun $\mathrm{N}$-terminal kinase 2 expression suppresses growth and induces apoptosis of human tumor cells in a p53-dependent manner. Mol. Cell. Biol. 20, 1713-1722.

Potapova, O., Haghighi, A., Bost, F., Liu, C., Birrer, M. J., Gjerset, R. and Mercola, D. (1997). The Jun kinase/stressactivated protein kinase pathway functions to regulate DNA repair and inhibition of the pathway sensitizes tumor cells to cisplatin. J. Biol. Chem. 272, 14041-14044.

Sampath, D., Cortes, J., Estrov, Z., Du, M., Shi, Z., Andreeff, M., Gandhi, V. and Plunkett, W. (2006). Pharmacodynamics of cytarabine alone and in combination with 7-hydroxystaurosporine (UCN-01) in AML blasts in vitro and during a clinical trial. Blood 107, 2517-2524.

Sampath, D. and Plunkett, W. (2000). The role of c-Jun kinase in the apoptotic response to nucleoside analogue-induced DNA damage. Cancer Res. 60, 6408-6415.

Stadheim, T.A., Saluta, G.R. and Kucera, G.L. (2000). Role of c-Jun N-terminal kinase/p38 stress signaling in 1-beta-Darabinofuranosylcytosine-induced apoptosis. Biochem. Pharmacol. 59, 407-418.

Tashiro, E., Simizu, S., Takada, M., Umezawa, K. and Imoto, M. (1998). Caspase-3 activation is not responsible for vinblastine-induced $\mathrm{Bcl}-2$ phosphorylation and G2/M arrest in human small cell lung carcinoma Ms-1 cells. Jpn. J. Cancer Res. 89, 940-946.

Turnbull, K. J., Brown, B. L. and Dobson, P. R. (1999). Caspase-3-like activity is necessary but not sufficient for daunorubicin-induced apoptosis in Jurkat human lymphoblastic leukemia cells. Leukemia 13, 1056-1061.

Weston, C. R. and Davis, R. J. (2007). The JNK signal transduction pathway. Curr. Opin. Cell. Biol. 19, 142-149.

Wisdom, R., Johnson, R. S. and Moore, C. (1999). c-Jun regulates cell cycle progression and apoptosis by distinct mechanisms. EMBO J. 18, 188-197.

Yu, R., Shtil, A. A., Tan, T. H., Roninson, I. B. and Kong, A. N. (1996). Adriamycin activates c-jun N-terminal kinase in human leukemia cells: a relevance to apoptosis. Cancer Lett. 107, 73-81. 